\title{
The Meaning of Arrows: Diagrams and Other Facets in System Sciences Literature
}

\author{
Jeffrey V. Nickerson \\ Stevens Institute of Technology \\ jnickerson@stevens.edu
}

\begin{abstract}
Understanding the use of diagrams and other components of scholarly systems-related papers may inform us about the papers, the field, and the way we think. We analyze 495 papers containing 1899 figures in a systems conference proceedings. Diagrams can be classified according to hard and soft genres. Most diagrams were found to be in soft genres. In combination, diagrams and other facet information can be used to classify papers - for example, a paper with a model diagram and a set of numeric tables will tend to be a statistical paper. The work has implications for automated document searching. The practical implication for authors is that more explicit consideration of diagram types might both clarify thinking and make later searching by other researchers easier. The implication for publishers is that collecting facet information, and presenting thumbnails, may enhance search for the research community.
\end{abstract}

\section{Introduction}

Diagrams are an integral part of systems literature. This follows from the nature of systems disciplines; systems are connected things; we study systems in the belief that we can abstract ideas from one system and apply them to others. Diagrams are one way we represent this abstraction - when we teach, and when we write scholarly papers.

In the reading activity that is part of our research, some of us seek out diagrams, and even choose which papers to read on the basis of the diagrams contained within. When journals and proceedings are in paper form, this skimming activity is easy - but as more papers and proceedings are published electronically, and as the rate of literature growth increases, we find that we rely more on search engines to find which papers we will even glance it. And the current state of search engines does not provide an easy way of finding papers with a certain sort of diagram.
In order to motivate the later discussion, consider the following search scenarios, derived from the author's recent experience; imagine a researcher looking for:

1. Papers containing layer diagrams which include a messaging subsystem.

2. Papers with models for researching issues of trust.

3. Empirical studies related to design education.

4. Images which show user interfaces for emergency response simulators.

5. Papers with proofs related to the tractability of bicriterion shortest-path problems.

In all cases, a keyword search helps find papers in the right domain. But the searches are really related to genre - the researcher is looking for particular types of papers, diagrams, images, or equations.

Genre studies go back to Aristotle [1]; the idea has been more recently discussed in a growing literature [26]. Crowston and Kwasnik propose using facets to define genres of documents [7]. They observe that users pay attention to particular clues to identify genres, including tables and figures. They note that one possible facet of a scholarly paper may be the graphics contained within.

In this paper, we develop further the observation that figures and tables can give clues to the genre of a paper. We think that diagrams have genres, and that these genres may correlate with the genre of the containing paper.

Our research program may, over time, have a larger significance. Diagrams may help us characterize papers. Papers in turn may help us characterize the conference tracks or journals they appear in. It may be that changes in research focus in a community manifest themselves in the changing nature of diagrams, so that analyzing diagrams may give us insights into entire fields of research.

Work in the taxonomy of diagrams has been ongoing for many years. Charles Pierce articulated a categorization of signs into icons, indices, and symbols that is still much used today; in the subcategory of indexical signs he discussed the diagram [8]. Nadin articulates the 
differences in configurational and sequential communication [9]. Bertin's work analyzes diagrams, including maps, charts, and what he refers to as networks [10]. Larkin and Simon discussed the relationship to computation and cognition [11]. Barbara Tversky, in collaboration with other researchers, has analyzed in depth the way we think with diagrams [12-16]; in particular, the analysis of charts and graphs by Zacks and Tversky has influenced this study [17].

\section{A model}

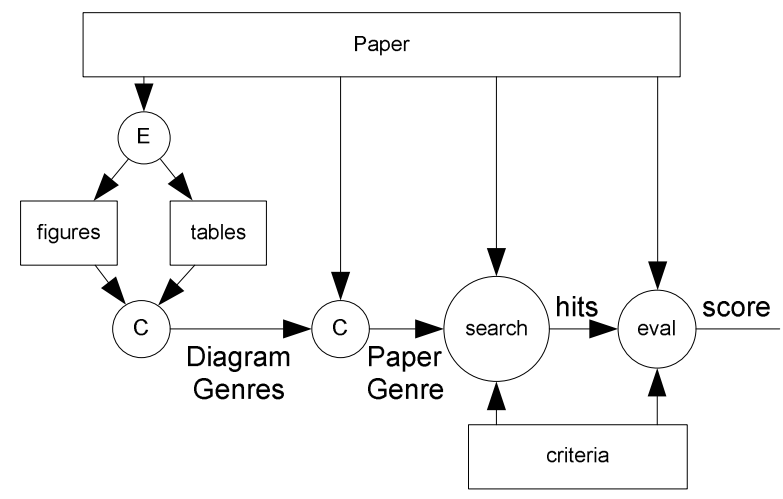

Figure 1. A model for the use of diagram classification

We have in mind a potential model for research in this area, shown in figure 1. This model is really the outgrowth of the exploratory study we are about to discuss; we present it first as we believe it makes the rest of the paper more intelligible.

A paper influences several processes. First, a paper feeds an extraction process, $E$, into figures and tables. These figures and tables go through a classification process, $C$, which in turn feeds another classification process to determine the genre characteristics of the containing paper. This feeds into a search process, in which multiple papers are compared. The success of the search can be evaluated in relation to specific search criteria. If studies such as this one suggest it might be useful, this model might be formally verified, and it may be that parts of the overall process could be automated. The presence of a score at the end of model may make it possible to evaluate ideas suggested here in comparison to other ideas. This paper focuses on one part of the model, the classification of tables and diagrams, in anticipation of more formal future studies.

We note also that this process may be operating to some extent already - that as skimmers of papers, we note diagrams and tables, and make our own genre classifications accordingly.

\section{Genres of scholarly papers}

Genres are readily identifiable categories, shared by convention. Genres are hierarchical, in two senses. First, scholarly papers are a genre of document, and there genres within the category scholarly paper. Second, documents may be composed from other component documents, and these component documents may have genres of their own.

What are the genres of scholarly papers? It depends on the field. A recent papers analyzes work in the computing disciplines [18]. Journal papers are categorized according to three major approaches: descriptive, evaluative, and formulative. Along a different axis, papers are categorized into 19 different methods. Several of these methods relate to our problem scenarios. For example, our interest in searching for empirical work might lead us to look for papers that use field studies, field experiments, data analysis, or laboratory experiment. Our interest in looking for a mathematical proof might lead us to look for a conceptual analysis/mathematical method. Finally, our interest in finding images of a prototype would lead us to look for a paper using the concept implementation (proof of concept) method.

If authors were to identify clearly the methods used in their papers, then searching for such papers would be easy. But they often don't. Papers may be a blend of different methods, may use no recognizable method, or may invent a new method. They may use an existing method, but never state the method in a manner that will be amenable to text search. In some fields, for example biology, there is a movement to produce results in a structured form so that all papers will be machine readable. In some medical fields, structured abstracts are the norm, and the research method is to be explicitly chosen from a set. Recently, one journal publisher, Emerald, has started a similar practice in the information systems field. Yet it is still an open question if or how we should classify genres, and at what stage in the publishing process.

\section{Method}

What we are doing can be thought of as an exploratory case study, as described in Yin [19]. We are starting with a set of general research questions. What is the relationship between components of a document, and the genre of a document? What are the relationships between the different facets of a paper? And can we classify the genre of paper components such as diagrams?

Within the context of the case study, we are performing data analysis. We analyze here all the 495 full papers in the digital Proceedings of the 37th Annual 
Hawaii International Conference on System Sciences [20].

We made two passes through the papers, first to make counts of the numbers of the components in the papers, then to extract the figures to separate files, which we printed. We categorized and counted the figures and captions isolated from the papers they were contained in. Finally, we performed some statistical tests on the data.

The author scanned all the papers and classified all the figures. In future work we plan to use multiple coders and to collect agreement statistics.

\section{Results}

\subsection{Components of scholarly papers}

Scholarly papers in the system sciences contain text, figures, tables, equations, captions, author lists, author affiliations, headings, references, an abstract, and optionally footnotes, acknowledgements, and appendices. These, or the aggregate statistics related to these, can all be seen as facets of the paper.

We counted the following, in looking at each paper: the number of authors, the number of references, the number of tables, the number of figures, and the number of equations. We found that tables and figures were easy to count. Equation counting was easy when authors separate them out and number them. But many authors embed the mathematics in the text, and it is hard to determine where one equation ends and another begins. As $80 \%$ of the papers have no equations at all, the presence of even one equation is useful information.

About 3\% of the papers had errors in the labeling of figures - figure numbers were skipped, or duplicated. In several cases, tables were mislabeled as figures. We decided to fix misclassifications and miscounts, in the anticipation that new reviewing systems will over time lessen the frequency of such errors.

\subsection{The major facets}

More than $99 \%$ of the papers have at least one equation, figure or table. On average, each paper contains 7 elements. One can see from figure 2 that the data points, representing individual papers, tend to cluster toward low numbers of the different elements; all the distributions are unimodal, but skewed toward 0 .

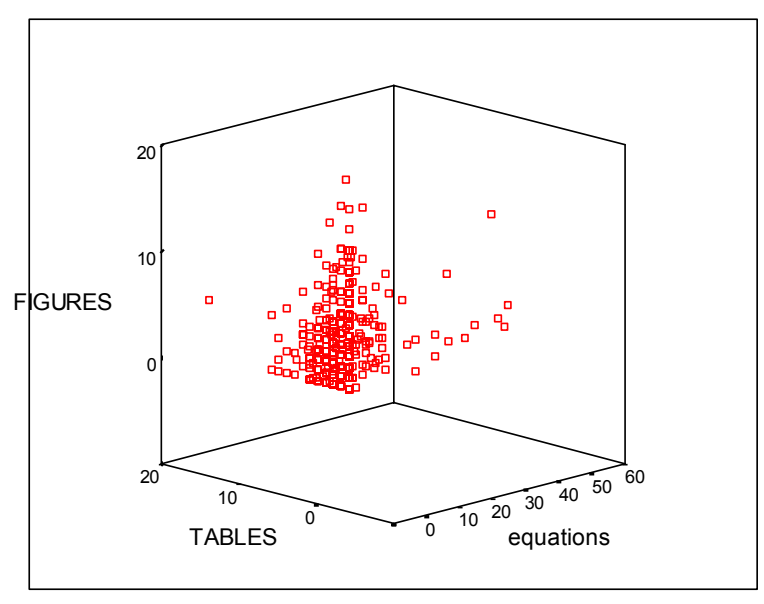

Figure 2. 3D scatter plot of figures, tables, and equations.

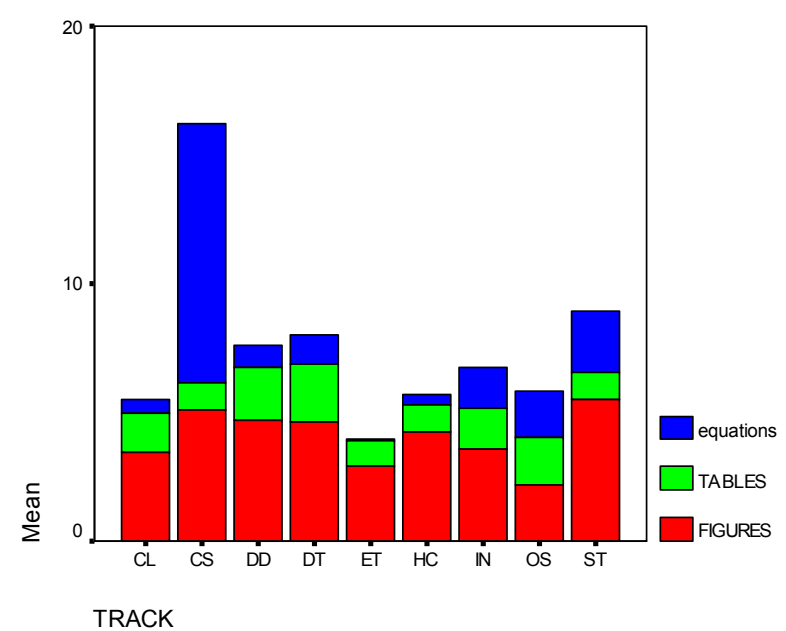

Figure 3. Equations, tables, and figures by track.

In looking at the mix by track, we see that the tracks differ. In particular, the complex systems track, which includes minitracks related to research on the electric grid, contains the most equations.

We can also look by the 84 minitracks, in Figure 4. The highest peak is the Electronic Commerce minitrack, which contains a concentration of economic papers. 


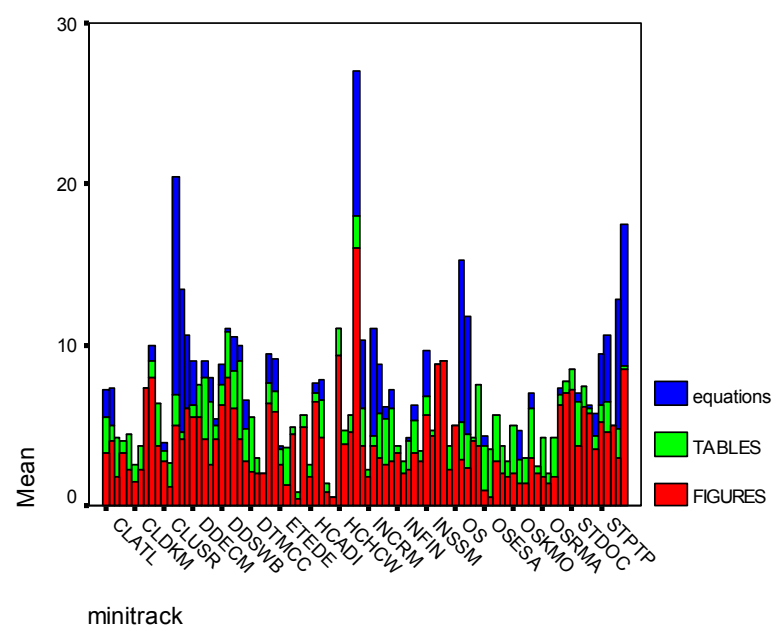

Figure 4. Equations, tables, and figures by minitrack.

In the spirit of exploratory data analysis, we looked at correlations across the facets we measured, shown in Table 1.

Table 1. Correlations using Kendall's Tau B. (Bold numbers are significant at the .01 level.)

\begin{tabular}{|l|l|l|l|l|}
\hline & Authors & Refs & Figures & Tables \\
\hline Refs & -.014 & & & \\
\hline Figures & $\mathbf{. 1 0 7}$ & $\mathbf{- . 2 4 3}$ & & \\
\hline Tables & .050 & .068 & $\mathbf{- . 1 5 2}$ & \\
\hline Equations & .034 & $\mathbf{- . 2 0 2}$ & .087 & .022 \\
\hline
\end{tabular}

What are we to make of the negative correlation between figures and tables? It could be that given space limitations, a lot of one precludes a lot of another.

And there is also a negative correlation between references and figures. One explanation is that there is only so much room in a paper - lots of figures means less text, and references might be proportional to text. However, if this were the case, one might expect a similar negative correlation between tables and references, which doesn't occur. One possible explanation is that the figure papers and table papers are written by different communities of researchers, with different norms for citation.

There seems to be interesting information just from a simple look at the facets. And now we proceed further, by breaking down the tables and figures into subtypes.

\subsection{Genres of tables}

Tables can contain textual information or numeric information. We counted 794 tables; 61\% contained numeric information. 170 papers, or $34 \%$, contain at least one numeric table. Why do we differentiate between numeric and textual tables? We noticed that the statistical, social science-based papers tended to use numeric tables to display the results of correlations and other analysis.

\subsection{Genres of figures}

Figures were easily classified into several types. We noted many screenshots. Also, many photographs, several maps, and several realistic computer-generated images. All of these we can clump together; note this is consistent with Peirce's first category of signs, the iconic, the signs that look like what they represent. Photographs, screenshots and realistic computer graphics can be easily detected, as they are displayed in raster, as opposed to vector, formats. Maps may be either raster or vector, and can range from super-realistic aerial images to more abstract, chart-like representations. (Maps are arguably a form of chart - in any event, they constitute less than $1 \%$ of the figures).

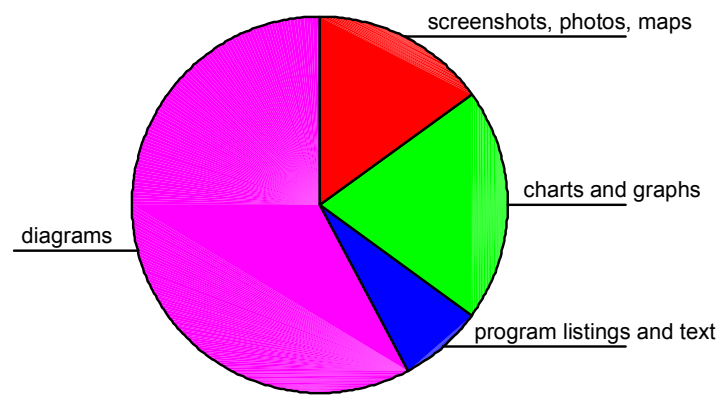

Figure 5. Types of figures

Next we can distinguish charts and graphs. Distinct from charts and graphs are topological figures showing abstract relationships. We call these diagrams. Note that the definitions of diagrams, charts, and graphs are not standardized; arguably charts and graphs are diagrams, and Bertin [10] thinks they are the primary diagrams - he goes so far as to put what we call diagrams into another category - networks.

Most important for this study is that for the discussion that follows the paper remains internally consistent. Charts and graphs are important to distinguish, as they usually establish that the paper is using some form of statistical or experimental method. Charts and graphs can be further broken down into subtypes, and other authors have done this $[10,17]$. 
We do not go as far as these authors; instead, we make a simple distinction. We separate graphs into the ones that display data points, plots, the ones that are more conceptual and guess at possible relationships (we originally labeled these psuedo-plots, but found some were very useful), and the ones used in economic papers, which show the plots of idealized equations. Of the chart and graph category, most are clearly plots (53\%) and charts $(27 \%)$. The remaining $20 \%$ is evenly split between conceptual and economic plots.

Figure 5 shows the breakdown of the 1899 figures we analyzed.

These different classes are easy to distinguish. Screenshots and photos are pixel-based, not vector based. Charts and graphs are distinguished by axes. Program listings include code, and have a telltale indentation structure. Other text-based displays are also easy to distinguish.

\subsection{Genres of diagrams}

Distinguishing the genres of the 983 diagrams was more difficult. There are several categories of diagrams that are well known and tightly specified. For example, flow charts are always directed graphs, and these graphs often contain loops. The way we normally recognize them is through the telltale diamond shaped decision nodes.

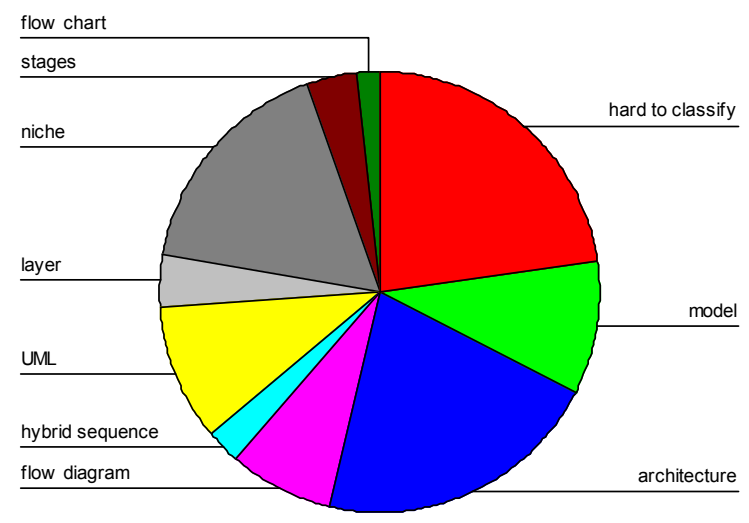

\section{Figure 6. Types of diagrams}

We listed out the recognizable types of diagrams which are tightly specified, and looked for them - we had over 20 of them. We were surprised to find that genres of diagrams that are tightly specified are far less common than loosely defined genres; of the 983 diagrams, only $37 \%$ are in tightly specified genres, such as those defined in the Unified Modeling Language (UML) standard [21].
The rest are in what we refer to as loosely specified genres - for example, the diagrams are captioned as an architecture or a model, but the formal characteristics will vary far more than with a flow chart. Another way of saying it is that there is no standard template for these loosely defined types.

Diagrams labeled as models show the relationship between dependent and independent variables. Sometimes the words influence diagram, research design, or framework are used in the caption instead of model. There were 101 of these diagrams in the papers. They are loose in that multiple conventions are used to differentiate and label the nodes and edges, although the norm is a directed graph read from left to right, from independent to dependent variables.

The most popular diagrams are architectural diagrams, showing how a system is put together. These are often labeled with architecture in the caption - but sometimes the words framework or structure are used. These diagrams often have undirected edges. Alternatively, the edges are bi-directional. There are 212 of these diagrams.

Related to this is a type we called a hybrid sequence diagram, in which architectural diagrams are labeled with numbers in such a way that a sequence of activities can be read off - there are 25 of these. Unlike the previous categories, there is no common way of referring to these diagrams - although they are a staple of system designers.

Another loose genre type is flow diagrams. There are 80 of these, and they usually indicate the flow of data between entities. Sometimes the architectural and flow diagrams are very close to each other. The general way to differentiate is to look at the arrows. Flow diagrams should have unidirectional arrows, and usually will not have cycles.

Layer diagrams are diagrams that look like layer cakes, as in protocol stacks. Sometimes these diagrams are labeled as layers, sometimes as stacks, sometimes as architectures.

We also found many diagrams that are designed to show the stages of either the research or a method - step 1 , followed by step 2. Sometimes these are drawn as cycles, sometimes as a sequence of boxes.

We have clumped together all the UML-defined software diagram types. Of these the most popular is the class diagram, followed by the sequence diagram. Interestingly, the UML class diagram seems to have supplanted the entity-relationship diagram in the literature.

For convenience in graphing, we include a category called niche. This is the aggregation of many wellunderstood tight-genre diagram types such as circuits, Venn diagrams, Block diagrams, network diagrams, and tree diagrams. 
There is a mass of diagrams that are hard to categorize: $19 \%$ of the overall diagrams in the conference.

\subsection{Mushy arrows}

Why are a large number of diagrams hard to classify? Key to understanding a diagram is knowing the meaning of the conventions used. And usually the problem is understanding the meaning of an arrow. For in the systems world, an arrow is used to indicate several ideas:

1. the next stage in a project

2. the next instruction in a program

3. the movement of a stream of data

4. the transmission of a message

5. influence

6. causation

7. physical movement in space

8. the direction of an abstract relation

9. something important to look at

In well-specified conventions, such as flow charts, we implicitly know that the arrow indicates the flow of control - technically, the positioning of a program counter to the next instruction. If we don't we are guided by explicit templates in our automated drawing tools. But in more loosely specified conventions, our implicit knowledge may be lacking.

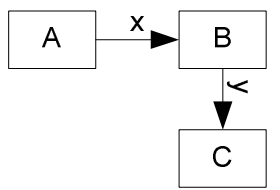

Figure 7. An ambiguous diagram.

For example, in one context, the diagram above could mean that A owns B, and B uses $\mathrm{C}$. It could also mean that $\mathrm{A}$ sends $\mathrm{B}$ an invoice, who passes it on to $\mathrm{C}$. The relative placement of the boxes may not mean anything or may indicate that process A precedes both $\mathrm{B}$ and $\mathrm{C}$, which run at the same time.

So, inadvertently, authors often overload the arrows an arrow will indicate the next stage in a process on one part of a diagram, and the transmission of a message on the other side. The general idea is that something is moving, but this is a vague and not very useful idea.

Most of the diagrams that are hard to classify have this feel - the arrows mean too many things at the same time, which makes it impossible to usefully classify them - and often to understand them. They have the look of meaning, but no meaning. While this might seem discouraging, remember that every paper has on average 7 elements, and multiple diagrams, so that paper-wide classifications can often still be made even if some diagrams are hard to understand.

Other hard-to-classify diagrams are imaginative blends of different types - future research may look at the way different conventions can be successfully combined. Finally, there are a small set of diagrams that appear to be coining new conventions - we found 19 of these, many in the area of mobile communication. New types of diagrams deserve attention, as they may embody new technical ideas or methods.

\subsection{Combinations}

What happens if we filter on combinations of different facets? For example, might not a combination of at least one model diagram and the presence of at least one numeric table indicate a statistical paper? We tried the filter - it yielded 32 papers. All except one are statistical papers. We find this result encouraging. It does not tell us how many statistical papers the filter missed - the false negatives - but it does suggest that such a filter would be useful in early searching by giving entry into the literature. Future research might confirm this finding, and look at both false positive and false negative error rates.

The exception, [22], is a case study. And it is not so far from what we were looking for - it is a case study with a model, and a mixture of qualitative and quantitative data. Perhaps we might find that case studies are likely to have models and mainly textual tables.

What about filtering on models in combination with graphs and charts? This yields 64 papers, only 8 of which are on the previous list. Most of the non-overlapping papers are different in nature from the first set; many are papers about the results of simulation, as opposed to field studies.

At first glance this doesn't seem to make sense - why shouldn't the genres be the same? For the choice of a graph or a table to represent data seems arbitrary. But it may not be. For example, to display multiple correlations, a table may be more efficient than multiple scatterplots. To display the comparison between a simulated and real data, plots may be most efficient. An alternative explanation is that certain disciplines may, by convention, favor certain genres of expression.

Table 2. Correlations using Kendall's Tau B. (All are significant at the .01 level)

\begin{tabular}{|l|l|l|}
\hline \multicolumn{1}{|c|}{ \#per paper } & Equations & Screen Shots \\
\hline Screen Shots & -.251 & \\
\hline Charts and Graphs & .254 & -.239 \\
\hline
\end{tabular}

Looking for correlations at a more specific level than those shown in table 1 , we examined the relationship 
between equations, screenshots, and our combined category of charts and graphs.

Table 2 shows that screenshots and equations are negatively correlated. But charts and graphs do correlate positively with equations. Charts and graphs are negatively correlated screen shots. There may be something related to genre going on. Papers with screenshots are often using a proof-of-concept method. Equations, charts and graphs are used in analytical, evaluative papers, and in formulative papers of a more quantitative bent.

We have not yet explored all possible combinations. A few may be particularly promising. To look for a prototype paper, we might look for a combination of screenshots and code listings. Just as individual diagrams seem to sometimes blend conventions, papers might blend conventions, and we might actually be able to formalize the extent of this blending, by examining the combinations of facets, consistent with the work of Fauconnier [23].

\section{Discussion}

\subsection{The search scenarios}

Looking back at the original search scenarios, can the information we gathered help in such searches?

The first search scenario is for layer diagrams. In our classification, we found that there are 40 of these diagrams in the conference. We also found that they are not universally captioned - some use the word layer, some the word stack, and others the more general terms architecture and framework. So text search may have difficulty finding them - we need some kind of diagram classification.

The second called for papers with models in them. We found lots of these, and our classification would help find them. Text search which could specify the word model to belong to a caption would work most, but not all, the time.

The third search looks for empirical papers. We have shown looking for numeric tables in combination with models works well.

The fourth looks for prototype images. We could search according to the number of screenshots.

Finally, a search for a mathematical proof will want to know that there are equations in the paper, and perhaps that there are also diagrams.

\subsection{Limitations}

This study is by its nature exploratory, and many different combinations of variables have been examined. In such situations, it is easy for experiment-wise error to occur. Over fitting to the data may have occurred, as well as unconscious anticipatory biasing in the categorizing of data. Any correlations or inferences in this study should be treated as preliminary. More formally defined followon studies may produce different results.

The study has looked at one sample set, a particular conference in a particular year. Subsequent studies should vary the year and the venue studied to assess the degree of generalizability of the results.

Since the fields involved are socially constructed, they are changing - the signals we perceive are not stationary, and this will limit our ability to predict. It may also be that observations about this or other conference, through this work or through unrelated work, might affect the behavior of future authors, either on their individual volition or through a changed review process.

\subsection{Research paradigms}

Some methods of research may be easier to distinguish than others, but this does not imply that such methods are somehow better or worse. For example, it is easier to pick out a statistical paper using the techniques here than an interpretive paper. In a different context, Kosaka has made a similar observation, and has urged those involved in interpretive research to come up with diagrammatic conventions [24]. He believes that such diagrams might ease the uninitiated reader's entry into the literature. But this paper suggests that diagrams might also be useful to the initiated reader, who might use their presence to aid in the search process. Of course, other techniques might also be used to make genre searching easier, and we are hesitant to suggest changes in established creative practices merely to aid in later search.

It could be argued that a too self-conscious approach to the use of facets in writing papers may produce more formulaic results. It can be counter-argued that we already implicitly understand these formulas. For example, professors sometimes coach graduate students to produce certain kinds of tables or diagrams early on in the creation of their papers - what the professors are doing is instructing students in how to produce the look of a particular genre - a necessary but not sufficient step.

\section{Implications for search}

If we find through repeated tests that analyzing the tables and figures of documents does aid us in searching, then what might be the implications for researchers and publishers?

There are three possible strategies that come to mind that might be used to take advantage of such a finding. 


\subsection{Author classification}

First, we might want to somehow classify writing prior to publication. For example, we might insist that authors self-annotate their work, choosing from a list of genres for their overall paper, and also from a list of genres for the tables and diagrams that they use.

While this might seem unlikely, we must remember that authors want to be cited, and that anything which increases the potential for being discovered through search engines may be seen as worth the effort.

Could the author be assisted in this process? Right now, much of electronic publishing works in a way that unintentionally strips out metadata. For example, an author may create a diagram using a tool, within which the author picks out a particular template. The template information is useful metadata. But this data is either lost when the diagram is saved, or lost later when the diagram is composed into a larger document. For example, many journals do not have the capability to handle all the formats of vector files, and ask that diagrams be converted into rasters prior to submission. But we think that vector data, and the metadata associated with the original creation of a diagram, may be useful information to embed in our electronic documents.

\subsection{Automatic Classification}

Second, we might decide to automatically attempt to detect and classify documents. We might want to do this out of a belief that author-dependent data collection will fail, or because we want to classify the backlog of already published literature.

How might we classify diagrams automatically? First of all, with the right tools, we might extract captions and use keywords in captions to infer the type of the diagram. This will work directly in many cases. In other cases, we might need to use text in the document to disambiguate caption terms that are too general, such as the ubiquitous term framework.

We might also analyze the diagram itself. If it is in vector form, our task is easier - we can then analyze the graph structure and the labels for clues as to the type of diagram. Formal graph properties correlate with types of diagrams - for example, some types of diagrams, such as flows, have directed edges, and are usually acyclic.

If the diagram is in raster form, we need to perform a raster-to-vector conversion and then analyze the vectors. This will introduce error [25]. We do not need a perfect conversion - we may need only to determine relatively easy features of the diagram, such as whether or not it has arrow heads, and whether these arrow heads are one or two-directional.
At a higher level, understanding diagrams is at least as hard as understanding text, so we expect that any automated attempt to classify diagrams will have high error rates. But even just a better-than-chance classification might contribute positively to the ranking of search results, and therefore be useful.

\subsection{Thumbnail-assisted search}

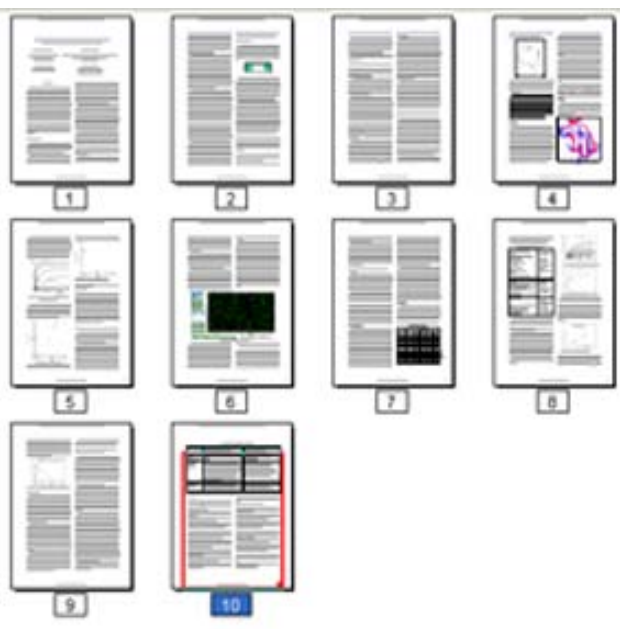

Figure 8. A thumbnail screenshot out of Adobe Acrobat for the conference paper DTMN05 [26].

In scanning the papers, Adobe Acrobat summary views proved valuable - we noticed it was possible to get a fairly good sense, and do a rough count of figures and tables, even from a very low resolution image such as that in figure 8. One can tell that there are tables, graphs, and diagrams. The thumbnail algorithm appears to be sophisticated enough to emphasize the tables and the graphs. However, one often can't tell from the thumbnail that there are equations in the paper, and one can't tell if the tables contain numbers or text.

In order to create more sophisticated thumbnails that would make equations visible, we might consider the work of Woodruff and others who have developed techniques to extract text terms and overlay them on document thumbnails [27, 28]. Using such a technique, we might extract a portion of an equation and overlay it on the thumbnails of papers which are math-intensive. Similarly, terms from tables might be extracted and overlaid to give a sample for the nature of the information contained.

Given such a technology, we might have an inexpensive alternative to the two previous classification methods. Instead of insisting on author self-categorizing, or running analyzers on all previous literature, we might simply serve up thumbnails of papers with the result of a text search. Since the thumbnails can be viewed faster 
than an abstract can be read, this approach may speed up our search result evaluation process.

Woodruff and others have observed that thumbnails allow us to assess genre quickly. Perhaps thumbnails work because they allow us to instinctively perceive facet characteristics, such as the ratio of diagrams and tables in a paper.

This leads one to ask if it might not be a good idea to include printed thumbnails in the books of abstracts distributed at large conferences. These thumbnails might provide a feel for the paper that would aid conference goers, allowing them the same kind of experience they might get by quickly looking through a full proceedings.

\section{Conclusions}

As scholarly literature becomes digital, we have lost our ability to flip through papers. Flipping can aid in search, as the components of a paper may be of interest in themselves, or may clearly indicate the research methods used.

In an exploratory data analysis, we have looked at the proceedings of a conference. We have reached several preliminary conclusions. First, the facets of a paper can be used to classify the paper. Second, that diagrams themselves can be classified according to genre, and this classification is useful.

The implications of the work are clear. We may want to collect more upfront information on papers and their facets in order to facilitate search. Alternatively, or in parallel, we may want to attempt to automatically recognize the genres of figures and tables. Finally, we may want to use the power of thumbnails to recreate the feeling - and the effectiveness - of flipping through the pages of a conference proceeding.

\section{Acknowledgements}

The author thanks his wife Deborah Morris for assistance in the extraction and analysis of the diagrams.

\section{References}

[1] Aristotle, Poetics. Mineola, N.Y.: Dover Publications, 1997.

[2] B. Kessler, G. Nunberg, and H. Schütze, "Automatic Detection of Genre," Proceedings of the 35th Annual Meeting of the Association for Computational Linguistics and the 8th Meeting of the European Chapter of the Association for Computational Linguistics, San Francisco CA, 1997.

[3] K. Crowston and M. Williams, "Reproduced and emergent genres of communication on the World-Wide
Web," The Information Society, vol. 16, pp. 201-216, 2000 .

[4] W. J. Orlikowski and J. Yates, "Genre repertoire: The structuring of communicative practices in organizations," Administrative Sciences Quarterly, vol. 33, pp. 541-574, 1994.

[5] E. G. Toms, D. G. Campbell, and R. Blades, "Does Genre Define the Shape of Information? The Role of Form and Function in User Interaction with Digital Documents," American Society for Information Science, 1999.

[6] T. Yoshioka, G. Herman, J. Yates, and W. Orlikowski, " Genre taxonomy: A knowledge repository of communicative actions," ACM Transactions on Information Systems (TOIS), vol. 19, no. 4, 2001.

[7] K. Crowston and B. H. Kwasnik, "A Framework for Creating a Facetted Classification for Genres: Addressing Issues of Multidimensionality," Proceedings of the 37th annual Hawaii International Conference on System Sciences, 2004.

[8] C. S. S. Peirce, Collected papers of Charles Sanders Peirce. Cambridge: Harvard University Press, 1931.

[9] M. Nadin, "On the meaning of the visual: 12 theses regarding the visual and its interpretation," Semiotica, vol. 52, no. 3/4, 1984.

[10] J. Bertin, Semiology of graphics. Madison, Wis.: University of Wisconsin Press, 1983.

[11] J. Larkin and H. Simon, "Why a diagram is (sometimes) worth ten thousand words," Cognitive Science, vol. 11, pp. 65-99, 1987.

[12] B. Tversky, "Cognitive origins of graphic conventions," in Understanding images, F. T. Marchese, Ed. New York: Springer-Verlag, 1995, pp. 29-53.

[13] B. Tversky, J. Zacks, P. Lee, and J. Heiser, "Lines, Blobs, Crosses and Arrows: Diagrammatic Communication with Schematic Figures," Diagrams 2000, 2000.

[14] M. Suwa and B. Tversky, " What do architects and students perceive in their design sketches? A protocol analysis," Design Studies, vol. 18, no. 4, pp. 385-403, 1997.

[15] M. Suwa and B. Tversky, "How do designers shift their focus of attention in their own sketches?," in Diagrammatic Representation and Reasoning, $\mathrm{M}$. Anderson, B. Meyer, and P. Olivier, Eds. London: Springer, 2001, pp. 241-254.

[16] M. Suwa and B. Tversky, "External representations contribute to the dynamic construction of ideas," in Diagrammatic Representation and Inference: Proceedings of Diagrams 2002, M. Hegarty, B. Meyer, and N. H. Narayanan, Eds. London: Springer, 2002, pp. 241-254.

[17] J. Zacks and B. Tversky, "Bars and Lines: A study of Graphic Communication," Memory and Cognition, vol. 27, pp. 1073-1079, 1999.

[18] R. L. Glass, V. Ramesh, and I. Vessey, "An analysis of research in computing disciplines," Communications of the ACM, vol. 47, no. 6, pp. 89-94, 2004.

[19] R. K. Yin, Case study research : design and methods, 3rd ed. Thousand Oaks, Calif.: Sage Publications, 2003.

[20] IEEE_Computer_Society, "Proceedings of the ThirtySeventh Annual Hawaii International Conference on 
System Sciences." Los Alamitos, Calif.: IEEE Computer Society, 2004, pp. CD-ROMs.

[21] J. Rumbaugh, I. Jacobson, and G. Booch, The unified modeling language reference manual, 2nd ed. Boston: Addison-Wesley, 2004.

[22] G. K. Roberts and J. B. Pick, "Technology Factors in Corporate Adoption of Mobile Cell Phones: A Case Study Analysis," Proceedings of the 37th Annual Hawaii International Conference on Systems Sciences, 2004.

[23] G. Fauconnier, Mappings in thought and language. Cambridge, U.K. ; New York, NY: Cambridge University Press, 1997.

[24] T. Kosaka, "The Notion of Diagrammatic Expression in Interpretive IS Research," Proceedings of the 6th PacificAsia Conference on Information Systems (PACIS), Tokyo, 2002.

[25] A. Chhabra and I. Phiillips, "The Second International Graphics Recognition Contest- Raster to Vector Conversion: A Report," in Graphics Recognition: Algorithms and Systems, Second International Workshop (volume 1389 of Lecture Notes in Computer Science), vol. 1389, K. Tombre and A. Chhabra, Eds. Berlin: Springer, 1998, pp. 390-410.

[26] W. W. Wakeland, E. J. Gallaher, L. M. Macovsky, and C. A. Aktipis, "A Comparison of System Dynamics and Agent-Based Simulation Applied to the Study of Cellular Receptor Dynamics," Proceedings of the 37th Annual Hawaii International Conference on Systems Sciences, 2004.

[27] A. Woodruff, R. Rosenholtz, J. B. Morrison, A. Faulring, and P. Pirolli, "A comparison of the use of text summaries, plain thumbnails, and enhanced thumbnails for Web search tasks," Journal of the American Society for Information Science and Technology, vol. 53, no. 2, 2002.

[28] A. Woodruff, A. Faulring, R. Rosenholtz, J. Morrsion, and P. Pirolli, "Using thumbnails to search the Web," Conference on Human Factors in Computing Systems archive, Proceedings of the SIGCHI conference on Human factors in computing systems, Seattle, Washington, United States, 2001. 